\title{
弱监督学习下的融合注意力机制的表面缺陷检测
}

\author{
孙美君，吕超章，韩亚洪*，李森，王征 \\ (天津大学智能与计算学部 天津 300350) \\ (yahong@tju.edu.cn)
}

\begin{abstract}
摘 要: 现有基于深度学习的缺陷检测方法通常采用强监督学习策略, 检测效果依赖于样本的数量和标注的质量. 针对上述问题，提出弱监督学习下融合注意力机制的神经网络算法，仅使用图像级别标签便可同时预测缺陷的位置 和概率. 首先对多尺度感受野模块提取的特征应用特征融合网络, 获取更多边缘细节信息; 然后通过多层次的自编 码器挖掘特征的深层语义信息; 同时通过三线性全局注意力模块进一步细化浅层特征的空间位置信息; 最后对浅层 边缘特征和深层语义特征进行融合增强, 得到最终的精细缺陷特征, 达到高效准确的自动化表面缺陷检测的目的. 基于 PyTorch 框架用 KolektorSDD 电转向器表面缺陷数据集验证所提算法, 并与 U-Net 等缺陷检测算法进行对比. 检 测视觉效果显示, 所提算法可以保留更多的细节纹理信息, 能够有效扩大细微缺陷与复杂背景之间的特征差异. 通 过大量实验表明, 该算法在复杂场景下比其他模型更为准确, 其精准率、 $F_{1}$ 值和总体精度都有所提升.
\end{abstract}

关键词: 深度学习; 表面缺陷检测; 弱监督学习; 注意力机制

中图法分类号: TP391.41 DOI: 10.3724/SP.J.1089.2021.18594

\section{Weakly Supervised Surface Defect Detection Based on Attention Mechanism}

\author{
Sun Meijun, Lyu Chaozhang, Han Yahong ${ }^{*}$, Li Sen, and Wang Zheng \\ (College of Intelligence and Computing, Tianjin University, Tianjin 300350)
}

\begin{abstract}
Nowadays, many defect detection algorithms based on deep learning are trained using a supervised learning strategy, which largely depends on the number of required samples and the quality of annotations. A weakly supervised attention network for surface defect detection is proposed, named SDD-Net, which can simultaneously predict both the location and probability of defects only by using image-level labels. Firstly, the feature fusion module is used to extract multi-scale edges features from the output of the multi-scale receptive field. Then, the deep semantic information of features is mined by multilevel auto-coder. Meanwhile, the trilinear global context attention module is used to further refine the spatial location information of shallow features. Finally, the SDD-Net is used to integrate the shallow edge features and deep semantic features to obtain the final fine defect features. The results of evaluation on KolektorSDD dataset demonstrate that the SDD-Net based on the PyTorch framework has better detection performance than other detection methods such as U-Net. It can retain more detailed texture information and effectively expand the feature difference between small defects and complex background. The experimental results show that the proposed model is more accurate than the other models in the complex scene, the precision, the $F_{1}$-score and the classification accuracy are significantly improved.
\end{abstract}

收稿日期: 2020-08-20; 修回日期: 2020-12-15. 基金项目：国家自然科学基金(61772360, 61876125). 孙美君(1978一), 女，博士， 教授, 博士生导师, CCF 会员, 主要研究方向为智能医疗影像; 吕超章(1996-), 男, 硕士研究生, 主要研究方向为缺陷检测; 韩亚洪 (1977一), 男, 博士, 教授, 博士生导师, CCF 高级会员, 论文通讯作者, 主要研究方向为多媒体分析与检索; 李森(1974一), 男, 硕 士, 工程师, 主要研究方向为数据库; 王征(1980-), 男, 博士, 教授, 硕士生导师, CCF 会员, 主要研究方向为视频显著性. 
Key words: deep learning; surface defect detection; weak supervision; attention mechanism

在产品制造工业化过程中，可能因某些不可 抗因素导致产品表面缺陷出现问题. 因此, 表面缺 陷检测是保证产品质量的必要任务.

采用传统的人工方法检测产品表面，耗费大量 人力、物力资源, 费时且效率低下; 并且人工处理 具有较强的主观性, 可能降低产品检测的准确性.

在经典的机器视觉方法中, 研究者首先采用 手工方式在特定产品领域中提取缺陷特征, 然后 使用基于规则或学习的分类器进行缺陷检测, 如 $K$ 近邻、支持向量机(support vector machine, SVM) 和决策树等. 但这些方法的性能在很大程度上取 决于手工设计方式所提取的特征的好坏。随着“工 业 4.0”和“中国制造 2025”的提出以及制造业数字 化、网络化、智能化的出现, 上述方法需要较长的 开发周期去适应不同的产品线周期，缺陷检测的 性能受到极大影响.

近年来，深度学习方法在图像领域的相关任 务中 ${ }^{[1-3]}$ 得到成功应用. 与经典的机器学习方法相 比, 深度学习方法能自动学习并挖掘图像数据中 具有代表性的特征, 并取得较好的检测效果. 然 而, 深度学习方法是一种数据驱动技术, 往往需要 大量的标注数据才能发挥作用. 但在实际生产中, 样本数据量少, 且难以获得. 因此, 如何在工业化 生产过程中利用少量数据训练并实现高性能分类 器是一项十分具有挑战性的任务.

为解决上述问题，本文提出一种基于弱监督 语义分割方法和注意力机制的工业表面缺陷检测 算法. 该算法不仅不需要强监督语义分割方法中 大量的信息标注，而且解决了传统深度卷积神经 网络中对于工业背景复杂下缺陷不敏感的问题; 同时, 采用批处理数据均衡策略减轻了样本数据 分布不平衡的问题，并用一张灰度图可视化缺陷 的潜在位置.

\section{1 相关工作}

\section{$1.1 工$ 工业缺陷检测}

早期，传统的缺陷异常检测方法遵循机器学 习的流程. 针对特定的问题领域, 使用人工方法对 图像特征进行设计并提取, 通常是用各种滤波器 组、直方图、小波变换、形态学算子、边缘检测等 技术. 但缺陷的多样性导致上述方法很难对缺陷 特征建模迁移，复用性不强.
近年来，随着深度学习方法的涌现，如 AlexNet ${ }^{[4]}, \mathrm{VGG}^{[5]}$, InceptionNet ${ }^{[6]}$, DenseNet ${ }^{[7]}$ 等, 深度学习方法在特征提取和检测分类上取得了非 常好的效果, 越来越多的研究者和工程人员开始 将深度学习方法引入缺陷检测中, 并取得了一定 的成果 ${ }^{[8-9]}$. 但为了保证模型具有较好的性能, 深 度学习对数据集有所要求: 一是用于训练和测试 的数据集要确保不同类别下样本数据分布相差不 大, 满足独立同分布条件; 二是要求训练样本量要 足够大. 但在实际的工业产品表面缺陷检测中这 2 个条件很难满足, 达不到现有神经网络方法训练 的要求. 因此, 如何在一个小规模缺陷检测数据上 训练一个高性能的神经网络模型, 是一个函待解 决的小样本问题. 如何在保证不降低模型性能的 前提下，显著地减少样本标注成本是另一项有挑 战性的工作.

\section{2 小样本问题}

表面缺陷检测中的关键问题是缺陷训练样本 缺乏. 因此, 如何有效地利用有限的训练样本得到 高性能的模型是本文的一个核心问题. 目前主要 有以下 3 种不同的解决方式. 一是通过缺陷数据样 本扩增的方法 ${ }^{[10]}$ 解决数据量不足的问题, 即通过 在原始的小数据集中引人数据增强操作或缺陷样 本合成 ${ }^{[11]}$ 的方式获得更多的训练样本, 但该方法 易对缺陷数据造成干扰. 二是迁移学习方式, Yang 等 ${ }^{[12]}$ 通过迁移学习的方法降低深度神经网络训练 的数据量要求, 其将模型在大型公共图像数据集 进行训练之后, 在相对较小的缺陷数据集上进行 优化. 但由数据集不足引起的过拟合问题并没有 完全得到解决, 并且用非工业数据集预先训练的 模型在复杂的工业环境下适应性有限. 三是设计 合理的网络结构, 也可以大大减少训练样本的需 求. Tabernik 等 ${ }^{[13]}$ 提出一种基于语义分割的缺陷检 测算法, 其仅需要少量的缺陷图像样本对模型进 行训练, 并通过分割网络提高决策网络的准确率. 为实现复杂背景下裂纹的有效检测, 王森等 ${ }^{[14]}$ 也 将全卷积神经网络引人图像裂纹检测中，实验结 果表明该方法有效地降低了误检率.

\section{3 弱监督学习}

上述解决小样本问题的方法采取的是有监督 的学习策略, 即训练过程需要像素级别标签, 人工 逐像素进行标注, 这为有监督学习下的表面缺陷 检测的实际应用提升了难度. 而基于无监督学习 
的检测方法难以辨认工业背景复杂下的缺陷. 例 如, Mei 等 ${ }^{[15]}$ 提出了一种基于卷积自编码器和图像 金字塔的无监督方法，其在布匹丝织物数据集上 效果很好，但在金属表面等数据集上效果一般，远 远达不到工业应用要求. 因此, 如何在不降低模型 性能的前提下，显著地降低缺陷数据标注的工作 量, 也是目前亟须解决的重要问题之一. 针对上述 问题, 比较有效的解决方法是采用弱监督学习, 它 主要分为 3 种类型: 不完全监督、不确切监督和不 精确监督. 主动学习技术是解决不完全监督问题 的技术之一, Feng 等 ${ }^{[16]}$ 借鉴 ResNet 结构并结合主 动学习的思想设计了一种用于缺陷检测的主动学 习框架, 并将其用于马路和建筑等裂纹检测中. 半 监督方法是解决不完全监督问题的另外一种技术. Akcay 等 ${ }^{[17]}$ 在一个单类、半监督学习范式的空间 中, 利用生成对抗网络, 联合高维图像空间的生成 和潜在空间的推理，提出了一种异常检测网络，实 验结果表明了该模型的有效性. 此外, Zhao 等 ${ }^{[18]}$ 提出了一种基于正样本训练的缺陷检测框架，通 过重建网络修复样本中的缺陷区域, 对比输人样 本和重建样本后得到缺陷区域，但如果背景过于 复杂, 该网络重建和修复图像还是很困难的. 不精 确监督是由人工标注时因认知错误或失误而标注 的标签, 需要对潜在的错误样本进行修正. 不确切 监督是指在训练中只给出粗粒度标签的一类学习 方法, 如在缺陷检测当中仅给出图片级别标签, 不 提供像素级别标签.

\section{4 注意力机制}

虽然弱监督学习能有效地减少样本标注工作 量, 但还是存在一个关键问题: 如何基于弱监督学 习方法得到待检测物体中缺陷的有效位置和形状 信息. 解决上述问题经典且有效的方法就是注意 力机制. Wang 等 ${ }^{[19]}$ 提出自注意力机制对图像中的 远程依赖建模，但该方法计算量大，耗费大量计算 资源. $\mathrm{Hu}$ 等 ${ }^{[20]}$ 提出的 SE block 主要是对不同通道 的权重重新标定并赋予其不同的权重，缺点是没 有充分利用全局上下文信息. Woo 等 ${ }^{[21]}$ 将注意力 机制分为通道注意力机制和空间注意力机制, 具 备即插即用的特点，但涉及了手动设计池化等复 杂操作. 王一鸣等 ${ }^{[22]}$ 已通过实验证明, 加人视觉 注意力模块后，图像中的缺陷区域特征被赋予更 高的权重，可以有效地提升缺陷检测的准确率.

针对上述问题，本文提出了聚焦于小数据的 弱监督的注意力网络. 首先, 为了缓解数据样本不 足的问题，对自编码网络进行改进并融合三线性
全局注意力模块, 用于逐像素的缺陷定位任务中. 将图像级别分类任务变为像素级别分类任务, 其 定位结果可用于辅助检测最终的分类结果，也可 用于分类结果的可视化解释. 除此之外, 为了减少 对标注数据的依赖, 训练过程仅使用图像级别标 签, 标注成本低且易获取. 本文致力于基于弱监督 学习方法检测物体的缺陷位置和形状信息. 所提 出的检测网络使用实际工业产品表面数据集进行 训练和测试, 实验证明, 模型通过弱监督学习训练 后可以有效地定位到缺陷位置.

\section{2 本文算法}

为实现对工业产品表面缺陷的有效检测，本 文提出弱监督学习下融合注意力机制的表面缺陷 检测网络(surface defect detect network, SDD-Net). 如图 1 所示, SDD-Net 由 4 个部分组成: 特征融合 模块(feature fusion module, FFM)、自编码模块 (autoencoder module)、三线性全局注意力模块 (trilinear global attention module, TLGAM)和决策 模块(decision module). SDD-Net 算法的特点具体 表现在以下 2 点：(1) 引人多尺度感受野模块 (multi-scale receptive field module, MRFM)扩展感 受野大小, FFM 将 MRFM 提取的不同层次特征进 行融合，获取更多缺陷的边缘细节信息; (2) 将 TLGAM 引人自编码模块, 使 SDD-Net 在进行特征 提取时更专注缺陷相关信息, 有效地扩大缺陷与 背景之间的特征差异.

\section{1 特征融合模块}

由于检测图像中缺陷尺寸差异较大，给缺陷 检测带来了困难. 因此, 为解决检测目标尺度不一 致问题, 本文提出了 MRFM. 其设计思路模拟了 人类视觉的感受野, 具有极强的特征提取能力. 为 了进一步提高 SDD-Net 捕捉浅层细粒度特征的能 力，将 MRFM 生成的不同层的特征映射通过 FFM 进行融合, 解决了因尺度不一致造成的缺陷检测 困难的问题.

FFM 结构细节如图 2 所示, 分别由上下 2 条不 同的支路组成，其模块输人为待检测图像 (尺寸为 $3 \times 1408 \times 512)$. 首先将输人图像送人下层支路的卷 积模块中进行处理，其流程如下：利用 2 个卷积核 为 $3 \times 3$ 、步长为 1 的卷积层和一个 $2 \times 2$ 的池化层 捕获待检测高分辨率图像中的细微缺陷. 需要注 意的是, 每个卷积层后都进行一次归一化操作 (batch normalization)和校正线性单元(rectified linear 


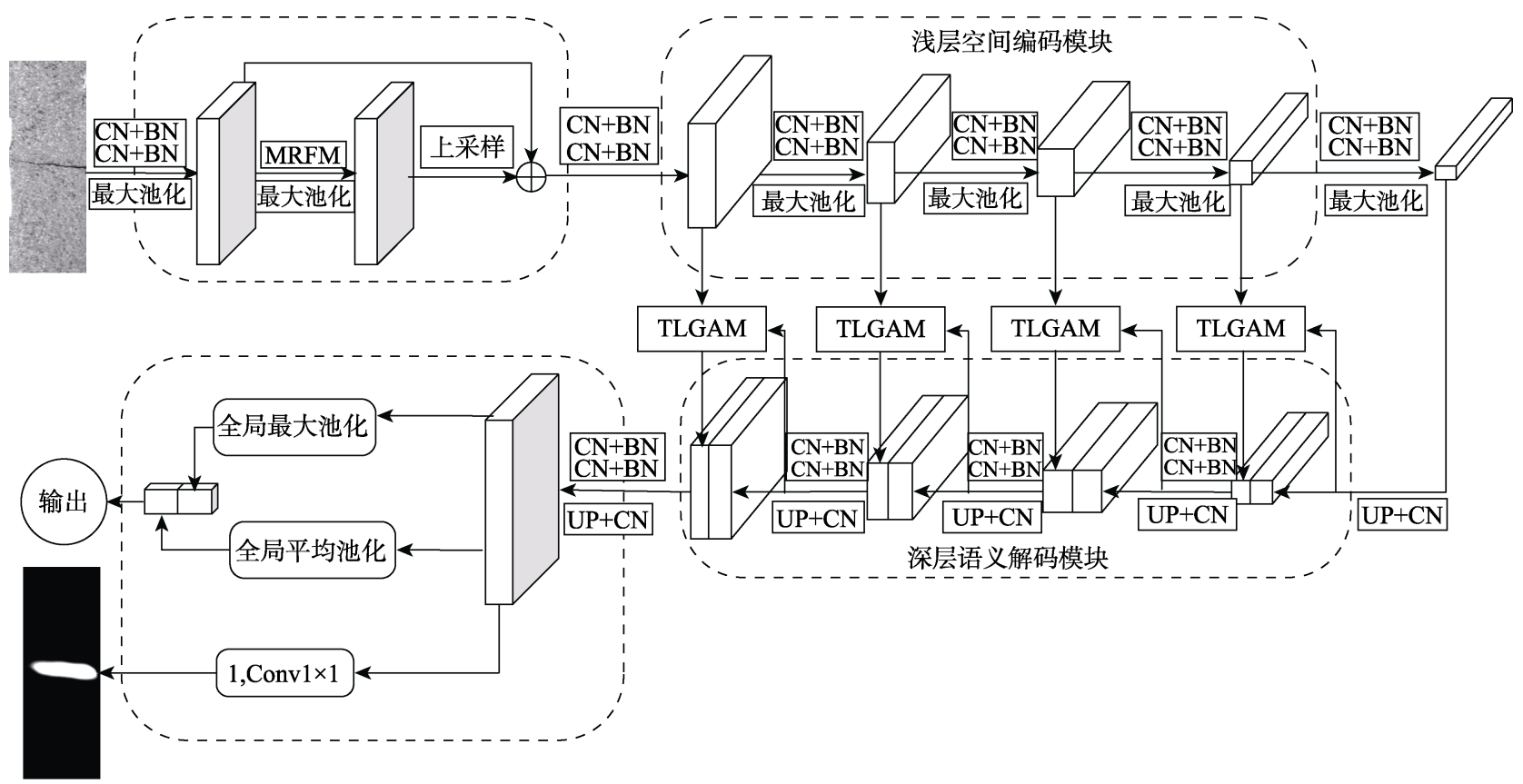

图 1 SDD-Net 结构示意图

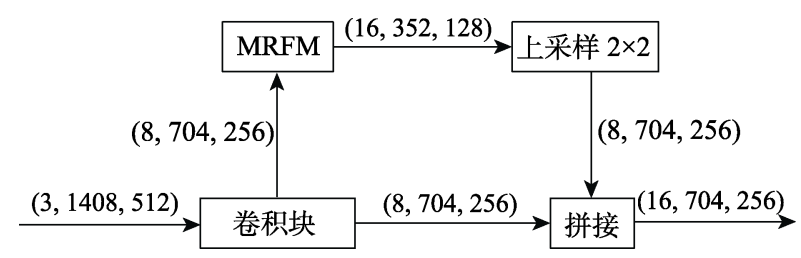

图 2 FFM 结构示意图

unit, ReLU)加快 SDD-Net 的收玫速度, 而池化层 的主要作用是减少模型参数量和计算量, 提高 SDD-Net 捕获图像中局部信息差异变化的能力.

接下来, 将下层支路卷积块输出的浅层特征 映射(shallow feature mapping, SFM)作为上层支路 MRFM 的输人. MRFM 采用不同卷积核的卷积操 作对输人的 SFM 进行处理, 最后将对应的结果相 加, 融合得到深层特征映射(deep feature mapping, DFM), 具体结构如图 3 所示. SFM 作为输人被送 人 MRFM 左右分支中: MRFM 的左侧分支首先使 用 $1 \times 1$ 的卷积操作对 SFM 在通道维度上降维, 该 操作不仅起到了降低计算量的作用，而且提高了 SDD-Net 对局部区域的感知能力. 之后卷积核大 小不同的卷积层构成了一个多分支并行结构, 并 对卷积结果进行了拼接. 这样做的好处主要是在 扩大了 MRFM 的感受野的同时, 进一步丰富了语 义信息; MRFM 的右侧分支不做任何处理，直接与 左侧分支的输出结果按一定比例进行求和操作, 得到 DFM. 该操作可以有效地加快网络训练的收 玫速度. 最后对 FFM 上下支路的输出使用拼接操 作，将 SFM 和 DFM 聚合. 由于待拼接的特征映射
尺度不同, 因此采用一个上采样操作将 DFM 的尺 度放大到与 SFM 相同的尺度, 这对于 FFM 获取丰 富的边缘细节信息至关重要.

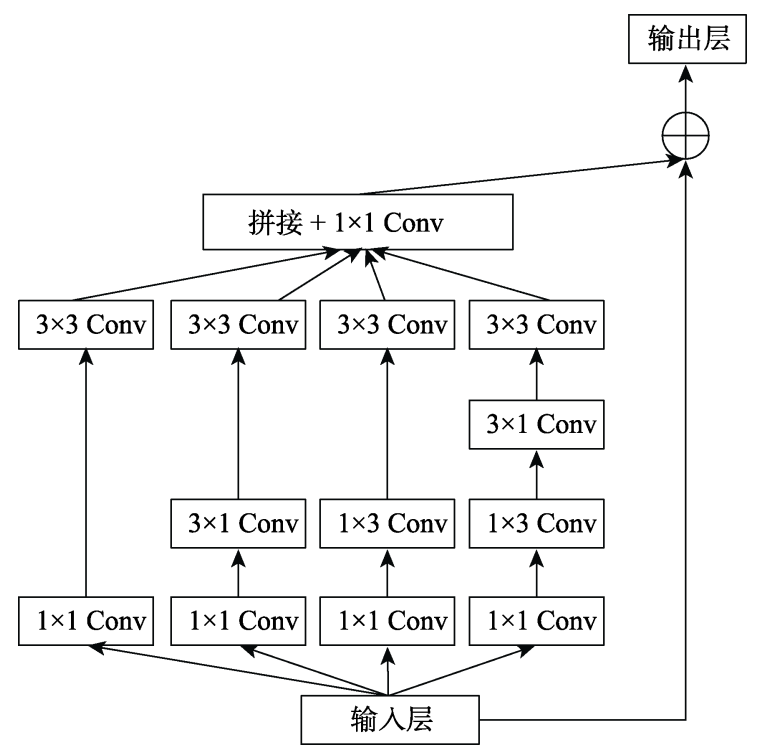

图 3 MRFM 结构示意图

\section{2 自编码模块}

自编码模块是最常见、最广泛的图像特征提取 工具. 具体设计细节如图 1 所示, 包含浅层空间编 码模块(shallow space encoder module, SSEM)和深 层语义解码模块 (deep semantic decoder module, DSDM)这 2 个重要组成部分.

在 SSEM 中对 FFM 生成的融合特征用卷积块 进行 4 次指定的操作. 其中, 卷积块由 2 个 $3 \times 3$ 的 
卷积操作以及一个 $2 \times 2$ 的池化操作构成. 在用卷积 块进行卷积操作时, 特征图的空间分辨率会逐渐 减小, 特征维度会逐渐增大. SSEM 通过一系列卷 积块操作可以层次式地学习到目标的浅层空间信 息，如边缘和位置信息.

在 DSDM 中, 对利用 SSEM 学习到的浅层空 间特征进行上采样操作，即通过 DSDM 逐层恢复 目标的空间分辨率信息. 在这个过程中，将 DSDM 中的深层特征进行上采样后, 与 SSEM 中的浅层 特征进行融合, 以便尽可能恢复缺陷的纹理信息. 并在深层和浅层特征融合的过程中引人 TLGAM, 对缺陷的细节特征进一步增强. 除此之外, TLGAM 的设计使 DSDM 能进一步学习到 SSEM 中丢失的相关特征, 从而弥补 DSDM 上采样过程 中损失的空间信息. 最后, 为学习到图像中具有判 别性区域的特征, 添加了 2 个 $3 \times 3$ 的卷积层, 进一
步增强了 DSDM 的语义学习能力.

\section{3 三线性全局注意力模块}

在深度神经网络中，应用注意力机制可以显 著提升模型的预测能力. 主要原因是注意力机制 为图像特征的各个通道赋予了不同的权重信息, 用来衡量不同特征通道的重要性，而这仅需增加 极少的参数量. 注意力机制的工作原理是为蕴含 高级语义信息的通道分配较大的权重, 为检测其 他无用特征的通道分配较小的权重, 使神经网络 能够很好地聚焦图像中更具判别性的区域. 本文 所提出的 TLGAM 受 $\mathrm{Li}$ 等 ${ }^{[23]}$ 启发, 用深层语义信 息指导浅层特征，并对浅层特征进行特征注意力 增强操作. 如图 4 所示, TLGAM 共分为 2 个分支, 第 1 个分支对浅层特征进行三线性增强操作，第 2 分支利用在深层特征中求得的权重信息进一步指 导增强后的浅层特征.

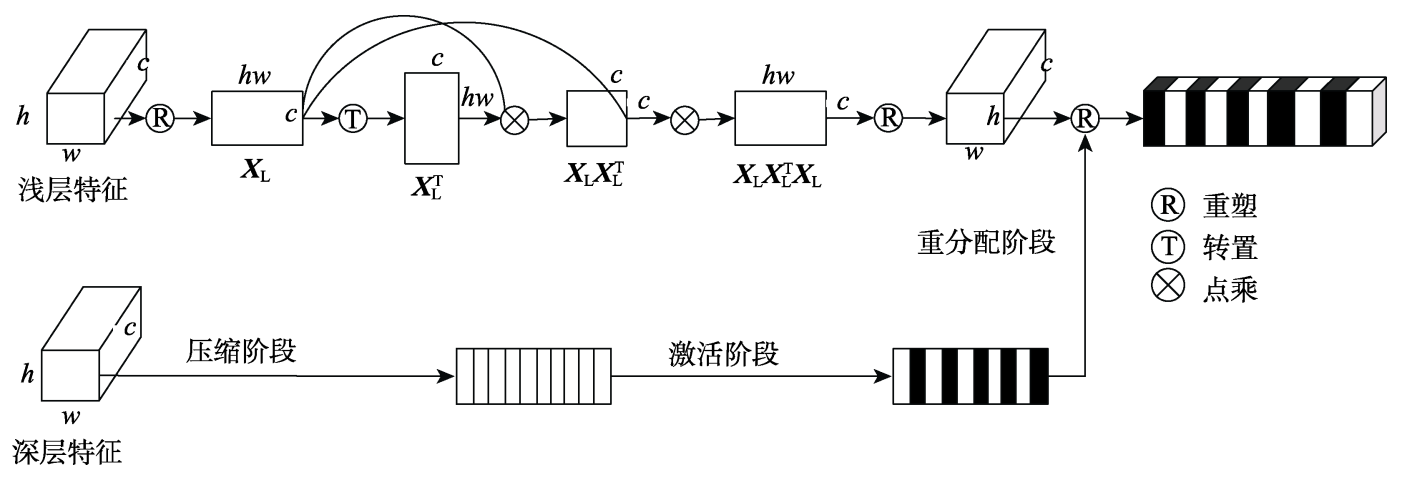

图 4 TLGAM 结构示意图

首先, 将 SSEM 中的浅层卷积特征图 $(c \times h \times w)$ 作为第 1 个分支的输人, 然后对输人特征图进行 reshape 操作变为 $\boldsymbol{X}_{\mathrm{L}}(c \times h w)$. 接下来进行转置操作 生成 $\boldsymbol{X}_{\mathrm{L}}^{\mathrm{T}}$, 将上述 2 步操作的结果相乘后得到通道 关系图 $\boldsymbol{X}_{\mathrm{L}} \cdot \boldsymbol{X}_{\mathrm{L}}^{\mathrm{T}}$, 通道关系图代表了浅层特征中各 通道之间的关系. 之后将上述通道关系图与 $\boldsymbol{X}_{\mathrm{L}}$ 再 作一次乘法操作得到 $\boldsymbol{X}_{\mathrm{L}} \cdot \boldsymbol{X}_{\mathrm{L}}^{\mathrm{T}} \cdot \boldsymbol{X}_{\mathrm{L}}$. 再经过 reshape 操作后，便可得到增强后的浅层特征

$$
\boldsymbol{A}_{\mathrm{L}}=R\left(N\left(N\left(\boldsymbol{X}_{\mathrm{L}} \cdot \boldsymbol{X}_{\mathrm{L}}{ }^{\mathrm{T}}\right) \cdot \boldsymbol{X}_{\mathrm{L}}\right)\right)
$$

其中， $R$ 表示 reshape 调整矩阵形状操作; $N$ 表示 Softmax 激活函数. 该分支可以方便、快速地将浅 层特征图转为注意力图, 起到了对浅层特征细节 部分增强的作用.

而在第 2 分支中，首先将 DSDM 中的卷积特 征图 $\boldsymbol{X}_{\mathrm{H}}$ 在空间维度上进行特征压缩; 之后通过激 励函数对特征通道进行激活, 得到通道相关性; 经 过上述 2 次全连接以及激活函数的操作后, 得到深
层特征的注意力图

$$
\boldsymbol{A}_{\mathrm{H}}=\sigma\left(F\left(\delta\left(F\left(\boldsymbol{X}_{\mathrm{H}}\right)\right)\right)\right)
$$

其中， $\sigma$ 表示 ReLU 激活函数; $\delta$ 表示 Sigmoid 激 活函数; $F$ 表示全连接函数.

在最后的指导阶段中，富含语义信息的深层 权重图会对增强后的浅层特征进行选择，即将深 层特征对应的权重值通过乘法操作逐通道加权到 已被增强的浅层特征图上，即

$$
\boldsymbol{X}_{\mathrm{o}}=F_{\mathrm{re}}\left(\boldsymbol{A}_{\mathrm{L}}, \boldsymbol{A}_{\mathrm{H}}\right)
$$

其中, $F_{\mathrm{re}}$ 为重分配函数.

\section{4 决策模块}

决策模块的输人特征是 DSDM 的输出特征, 该模块主要由 $1 \times 1$ 卷积、全局平均池化和全局最 大池化组成. 决策模块分为上下 2 条支路, 实现过 程如下：在下层支路中, 使用 $1 \times 1$ 的卷积操作对 DSDM 的输出特征图进行卷积, 得到缺陷的潜在 位置灰度图; 而在上层支路中，用全局最大池化和 
全局平均池化操作替代卷积神经网络中传统的全 连接层, 对 DSDM 的输出特征图进行相关操作. 其中，全局最大池化操作可以增强 SDD-Net 的平 移不变性, 从而提高了模型的预测能力; 全局平均 池化操作赋予了每个通道实际的内在意义. 经过 上述 2 步操作共生成 6 个输出神经元. 最后, 将上 述神经元通过 $1 \times 1$ 卷积操作以及 Sigmoid 激活函 数操作后, 输出待检测图像包含缺陷的概率.

\section{3 实验及结果}

\section{1 实验数据与评价标准}

为验证 SDD-Net 的有效性, 在 KolektorSDD 电子转向器的表面裂纹图像数据集 ${ }^{[13]}$ (简称 KolektorSDD 数据集)上进行了训练和评估. 该数 据集是在工业生产环境中采集而来的，每幅图像 分辨率都是 $1408 \times 512$ 像素, 如图 5 所示. KolektorSDD 数据集由 50 个有缺陷的电子转向器样本构 成, 每个转向器有 8 个表面, 共产生 399 幅图像及 其对应的 GT(ground truth). 其中 52 幅图像中有清 晰可见的缺陷，作为正样本; 其余 347 幅图像为无 缺陷图像, 作为负样本. 训练集中, 正样本数为 26 幅, 负样本为 224 幅; 测试集中, 正样本数为 26 幅, 负样本为 123 幅.

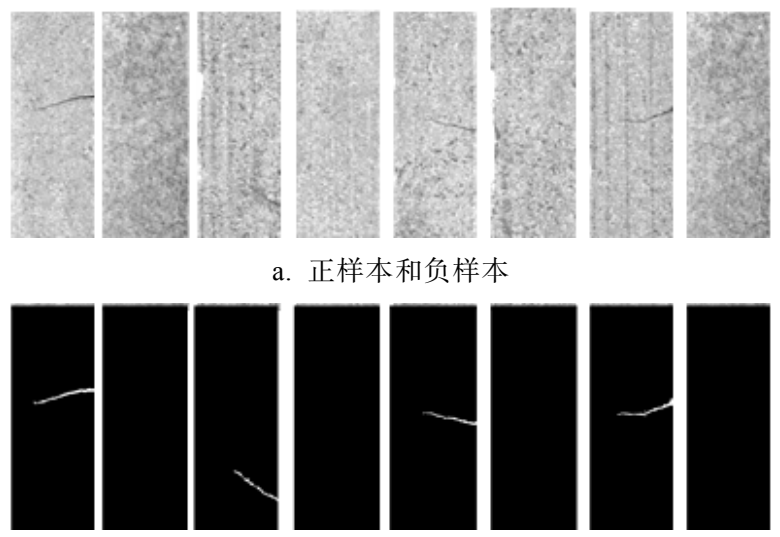

b. 像素级别标注标签

图 5 KolektorSDD 数据集

为验证模型的有效性，并证明其具有很好的 实用价值，实验选取精准率(precision， $P$ )、召回率 (recall， $R$ )， $F_{1}$ 值和准确率 (accuracy，A) 对缺陷 检测结果进行定量分析. 它们分别定义为

$$
\begin{gathered}
P=\frac{p_{i i}}{p_{i i}+p_{j i}} \\
R=\frac{p_{i i}}{p_{i i}+p_{i j}}
\end{gathered}
$$

$$
\begin{gathered}
F_{1}=2 \times \frac{P \times R}{P+R} \\
A=\frac{p_{i i}}{p_{i i}+p_{i j}+p_{j j}+p_{j i}}
\end{gathered}
$$

其中，在本实验中有 2 个分类：用 $i$ 表示有缺陷的 图像(正样本), 用 $j$ 表示无缺陷的图像(负样本); $p_{i i}$ 指属于类别 $i$ 被预测为 $i$ 的图像数量; $p_{i j}$ 指属 于类别 $i$ 被预测为 $j$ 的图像数量; $p_{j j}$ 属于类别 $j$ 被预测为 $j$ 的图像数量; $p_{j i}$ 属于类别 $j$ 被预测为 $i$ 的图像数量.

\section{2 实验细节}

SDD-Net 是用 Facebook 人工智能研究实验室 开发的 PyTorch 框架搭建的, 并用 Adam 算法优化 损失函数. 学习率大小设置为 0.0001 , 其中一阶动 量项为 0.5 , 二阶动量项为 0.999 , 共迭代 300 轮.

在训练和验证过程中, 由于 GPU 内存有限, 并且待检测图像尺寸较大, 因此在每轮迭代过程 中仅使用单幅图像，即批处理大小设置为 1 . 由于 无缺陷图像的数量是缺陷图像数量的 8 倍, 为了确 保网络训练过程中有缺陷的图像和无缺陷的图像 数量均衡, 采取了批处理数据均衡策略, 具体操作 为: 训练过程中, 在偶数迭代轮次时读取有缺陷的 图像, 在奇数迭代轮次时读取无缺陷的图像. 该策 略能够很好地分配缺陷图像和无缺陷图像, 缓解 了工业缺陷检测任务中样本不均衡的问题.

SDD-Net 的实验环境为: Ubuntu16.04 操作系 统, Python 3.7.4, 4 核 AMD Ryzen 1500X 处理器, 11 GB NVIDIA GetForce GTX 1080Ti 显卡.

\section{3 实验结果分析}

\subsubsection{KolektorSDD 数据集实验结果分析}

为评估 SDD-Net 的有效性，与当前常见的算 法包括 ResNet101 ${ }^{[1]}$, SE-ResNet101 ${ }^{[20]}$, SegNet ${ }^{[24]}$, U-Net ${ }^{[25]}$, DeepLabv3 ${ }^{[3]}$ 和 Seg and dec network ${ }^{[13]}$ 在 不同度量指标下进行对比. 从表 1 中可以看出, 本 文 SDD-Net 具有很好的分类性能, 在 KolektorSDD 数据集上的预测准确率达到了 $99.33 \%$, 而且参数 量少，网络收玫速度快。相比之下，分类网络 ResNet101 由于连续的卷积和池化操作, 所提取到 的特征不能很好地描述小样本数据集中的微小缺 陷, 分类性能一般. SE-ResNet101 与 ResNet101 结 构类似，不同之处在于 SE-ResNet101 中添加了注 意力模块, 分类性能比 ResNet101 有了一定程度的 提升，但精准率过低，仅有 $68.57 \%$. 然后在经典的 分割网络 SegNet, U-Net 和 DeepLabv3+上进行实 
验, 为保证实验结果只受网络结构的差异影响, 对 上述分割网络添加分类模块以实现最终的类别预 测. 其中 DeepLabv3+性能最佳, 在召回率为 $100.00 \%$ 的情况下，预测准确率为 $97.99 \%$. 而 Tabernik 等 ${ }^{[13]}$ 提出的基于强监督学习的 Seg and dec network, 具体流程为: 首先使用像素级别标签 对分割网络进行预训练, 然后在分割网络的基础 上搭建分类网络, 再次训练, 预测准确率可达 99.33\%. 然而，该方法需要像素级别标签的支撑， 耗时耗力. 与这些方法不同，本文提出的 SDD-Net 在训练样本较少的情况下, 仅需图像级别标签便 可实现优良的检测性能, 这与使用像素级别标签 的相关方法性能相当.

表 1 不同算法度量指标对比

\begin{tabular}{|c|c|c|c|c|}
\hline 算法 & $P$ & $R$ & $F_{1}$ & $A$ \\
\hline ResNet101 $1^{[1]}$ & 36.96 & 65.38 & 47.22 & 74.50 \\
\hline SE-ResNet101 ${ }^{[20]}$ & 68.57 & 92.31 & 78.69 & 91.28 \\
\hline $\mathrm{SegNet}^{[24]}$ & 92.31 & 92.31 & 92.31 & 97.32 \\
\hline U-Net ${ }^{[25]}$ & 92.59 & 96.15 & 94.34 & 97.99 \\
\hline DeepLabv3 $+^{[3]}$ & 89.66 & 100.00 & 94.55 & 97.99 \\
\hline Seg and dec network ${ }^{[13]}$ & 100.00 & 96.15 & 98.04 & 99.33 \\
\hline SDD-Net & 100.00 & 96.15 & 98.04 & 99.33 \\
\hline
\end{tabular}

\subsection{2 消融实验结果分析}

为验证 FFM 和 TLGAM 对 SDD-Net 整体性能 上的贡献，对 SDD-Net 进行拆分实验，分别验证 FFM 和 TLGAM 在模型中所起的作用, 测试结果 如表 2 所示. 实验结果表明，使用 FFM 和 TLGAM 可以有效滤除图像中工业生产环境的复杂背景, 生成清晰而完整的缺陷位置图，提升模型性能.

\begin{tabular}{lcc}
\multicolumn{1}{c}{ 表 2} & SDD-Net 算法拆分实验 & $\%$ \\
\hline \multicolumn{1}{c}{ 算法 } & $F_{1}$ & $A$ \\
\hline Encoder-Decoder Backbone & 84.44 & 95.30 \\
Backbone+FFM & 88.00 & 95.97 \\
Backbone+TLGAM & 92.00 & 97.32 \\
Backbone+FFM+TLGAM & 98.04 & 99.03 \\
\hline
\end{tabular}

\subsection{3 算法效率分析}

本文提出的 SDD-Net 与其他模型在参数量、 平均精度以及运行速度方面也进行了全方位对比 实验. 其中平均精度是精准召回率曲线下的计算 区域，其能够精确地表示不同阈值下的模型性能. 从表 3 和图 6 中可以看出, SDD-Net 在保证较高的
检测精度的同时兼具较快的检测速度，检测单幅 图片用时 $30 \mathrm{~ms}$. SDD-Net 参数量为 $3.54 \mathrm{M}$, 仅是 U-Net 参数量的一半. SDD-Net 检测速度较快的原 因是: SDD-Net 在提取特征时, 并未采用类似于用 VGG 作为主干网络的方法提取基础特征，而是使 用 FFM 生成更精细的特征, 使 SDD-Net 在保持良 好性能的同时大大降低了模型参数量.

表 3 不同算法参数量和平均精度的对比

\begin{tabular}{|c|c|c|}
\hline 算法 & $10^{-6} \times$ 参数量 & 平均精度 $/ \%$ \\
\hline ResNet101 ${ }^{[1]}$ & 42.50 & 74.50 \\
\hline SE-ResNet101 ${ }^{[20]}$ & 47.28 & 86.49 \\
\hline SegNet ${ }^{[24]}$ & 29.44 & 97.32 \\
\hline U-Net ${ }^{[25]}$ & 7.78 & 98.17 \\
\hline DeepLabv3 $+^{[3]}$ & 59.34 & 98.89 \\
\hline Seg and dec network ${ }^{[13]}$ & 15.47 & 99.66 \\
\hline SDD-Net & 3.54 & 99.79 \\
\hline
\end{tabular}

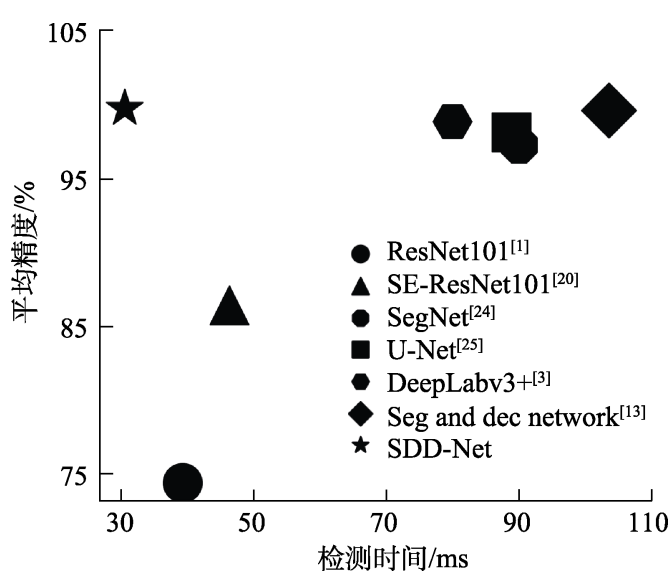

图 6 单幅图像平均精度下的检测时间

\subsection{4 可视化结果分析}

为了进一步证明 SDD-Net 模型性能的优越性, 对 SDD-Net 的预测结果进行了如图 7 所示可视化 输出实验. 需要注意的是, 结果图仅用做 SDD-Net 预测结果的可视化. 从图 7 中可以看出, 本文提出 的 FFM 和 TLGAM 有效地增强了缺陷像素和非缺 陷像素之间的差异. 除此之外, 测试时将 SDD-Net 模型进一步扩展到铁轨表面缺陷 RSDDs 数据集上, 如图 8 所示. 虽然待检测图片中有些缺陷区域肉眼 难以分辨, 但 SDD-Net 在无需像素级别标注标签 的前提下, 可以很好地聚焦到输人图像中的异常 区域, 能从图像级别标签中捕捉微小的异常特征, 在复杂的背景下感知裂纹细节。实验结果表明, SDD-Net 能够有效地检测到缺陷。 

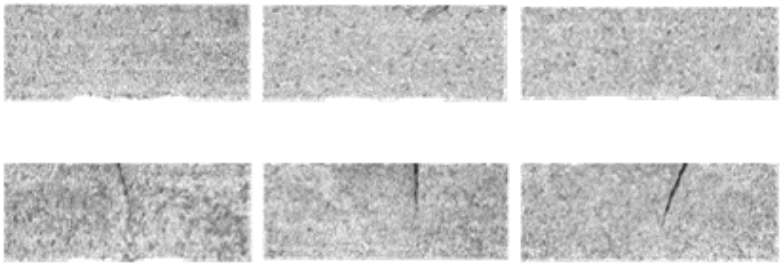

a. 原始图像
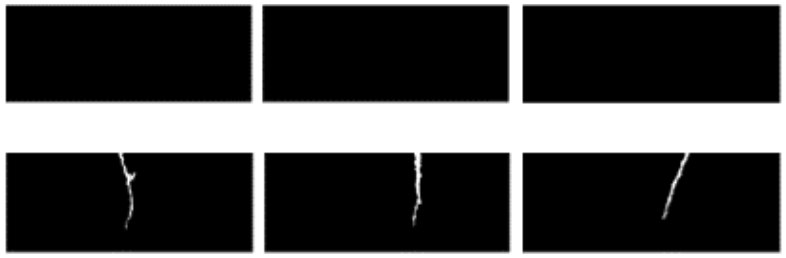

b. 像素级别标注标签
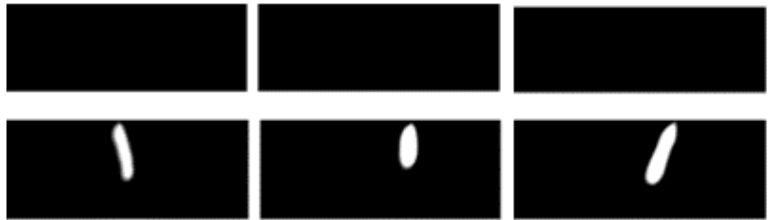

c. SDD-Net 输出缺陷潜在位置灰度图

图 7 KolektorSDD 数据集上的例子
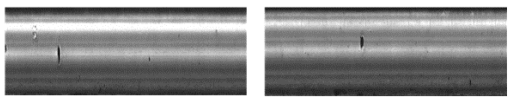

a. 原始图像
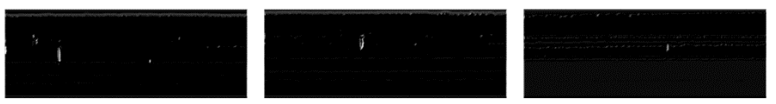

b. SDD-Net 输出缺陷潜在位置灰度图

图 8 RSDDs 数据集上的例子

\section{4 结 语}

本文提出了一种用于表面缺陷检测的弱监督 学习下的融合注意力机制的深度卷积神经网络架 构, 并在小样本的 KolektorSDD电机转向器表面裂 纹数据集上进行了测试. 该方法仅使用图像级别 标签便可同时预测缺陷的位置和检测概率，并获 得优异的检测性能. 实验结果表明, 该模型的准确 率与使用像素级别标签的其他方法性能相当. 本 文 SDD-Net 通过将浅层次的视觉特征和深层次的 语义特征有效融合, 并利用三线性全局注意力模 块, 能够有效地扩大缺陷像素和非缺陷像素之间 的差异, 使模型能够更精准地定位到图像中的缺 陷区域. 此外, 本文使用的批处理数据均衡策略在 一定程度上缓解了工业缺陷检测任务中样本分布 不均衡问题, 具有一定范围的普适性. SDD-Net 虽 然在 KolektorSDD 数据集上取得了较好的检测结
果, 但仍然有改进空间. 本文实验只在标注好的公 开数据集上进行了测试及对比, 但从理论方面来 看, SDD-Net 可以进一步用于识别其他材料(如钢 或玻璃)中的缺陷, 并尝试在其他的工业实际应用 中发挥价值.

\section{参考文献(References):}

[1] He K M, Zhang X Y, Ren S Q, et al. Deep residual learning for image recognition[C] //Proceedings of the IEEE Conference on Computer Vision and Pattern Recognition. Los Alamitos: IEEE Computer Society Press, 2016: 770-778

[2] Zhao Q J, Sheng T, Wang Y T, et al. M2Det: a single-shot object detector based on multi-level feature pyramid network[C] //Proceedings of the AAAI Conference on Artificial Intelligence. Palo Alto: AAAI Press, 2019: 9259-9266

[3] Chen L C, Zhu Y K, Papandreou G, et al. Encoder-decoder with atrous separable convolution for semantic image segmentation[C] //Proceedings of the European Conference on Computer Vision. Heidelberg: Springer, 2018: 833-851

[4] Krizhevsky A, Sutskever I, Hinton G E. ImageNet classification with deep convolutional neural networks[J]. Communications of the ACM, 2017, 60(6): 84-90

[5] Simonyan K, Zisserman A. Very deep convolutional networks for large-scale image recognition[OL]. [2020-08-20]. https:// arxiv.org/pdf/1409.1556.pdf

[6] Szegedy C, Ioffe S, Vanhoucke V, et al. Inception-v4, inception-ResNet and the impact of residual connections on learning[C] //Proceedings of the AAAI Conference on Artificial Intelligence. Palo Alto: AAAI Press, 2017: 4278-4284

[7] Huang G, Liu Z, van der Maaten L, et al. Densely connected convolutional networks[C] //Proceedings of the IEEE Conference on Computer Vision and Pattern Recognition. Los Alamitos: IEEE Computer Society Press, 2017: 2261-2269

[8] Faghih-Roohi S, Hajizadeh S, Núñez A, et al. Deep convolutional neural networks for detection of rail surface $\operatorname{defects}[\mathrm{C}] / /$ Proceedings of the International Joint Conference on Neural Networks. Los Alamitos: IEEE Computer Society Press, 2016: 2584-2589

[9] Yuan H, Chen H, Liu S W, et al. A deep convolutional neural network for detection of rail surface $\operatorname{defect}[\mathrm{C}] / /$ Proceedings of the IEEE Vehicle Power and Propulsion Conference. Los Alamitos: IEEE Computer Society Press, 2019: 1-4

[10] Yuan Gonglin, Hou Jing, Yin Kuiying. Night-time aerial image vehicle recognition technology based on transfer learning and image enhancement[J]. Journal of Computer-Aided Design \& Computer Graphics, 2019, 31(3): 467-473(in Chinese)

(袁功霖, 侯静, 尹奎英. 基于迁移学习与图像增强的夜间 航拍车辆识别方法[J]. 计算机辅助设计与图形学学报, 2019, 31(3): 467-473)

[11] Racki D, Tomazevic D, Skocaj D. A compact convolutional neural network for textured surface anomaly detection[C] // Proceedings of the IEEE Winter Conference on Applications of Computer Vision. Los Alamitos: IEEE Computer Society Press, 2018: 1331-1339 
[12] Yang H, Mei S, Song K Y, et al. Transfer-learning-based online Mura defect classification[J]. IEEE Transactions on Semiconductor Manufacturing, 2018, 31(1): 116-123

[13] Tabernik D, Šela S, Skvarč J, et al. Segmentation-based deep-learning approach for surface-defect detection[J]. Journal of Intelligent Manufacturing, 2020, 31(5): 759-776

[14] Wang Sen, Wu Xing, Zhang Yinhui, et al. Image crack detection with fully convolutional network based on deep learning[J]. Journal of Computer-Aided Design \& Computer Graphics, 2018, 30(5): 859-867(in Chinese)

(王森, 伍星, 张印辉, 等. 基于深度学习的全卷积网络图像 裂纹检测 $[\mathrm{J}]$. 计算机辅助设计与图形学学报, 2018, 30(5): 859-867)

[15] Mei S, Yang H, Yin Z P. An unsupervised-learning-based approach for automated defect inspection on textured surfaces[J]. IEEE Transactions on Instrumentation and Measurement, 2018, 67(6): 1266-1277

[16] Feng C, Liu M Y, Kao C C, et al. Deep active learning for civil infrastructure defect detection and classification[C] //Proceedings of the ASCE International Workshop on Computing in Civil Engineering. Seattle: American Society of Civil Engineers Press, 2017: 298-306

[17] Akcay S, Atapour-Abarghouei A, Breckon T P. GANomaly: semi-supervised anomaly detection via adversarial training[C]// Proceedings of the Asian Conference on Computer Vision. Heidelberg: Springer, 2018: 622-637

[18] Zhao Z X, Li B, Dong R, et al. A surface defect detection method based on positive samples[C] //Proceedings of the $\mathrm{Pa}$ cific Rim International Conference on Artificial Intelligence. Heidelberg: Springer, 2018: 473-481
[19] Wang X L, Girshick R, Gupta A, et al. Non-local neural networks[C] //Proceedings of the IEEE Conference on Computer Vision and Pattern Recognition. Los Alamitos: IEEE Computer Society Press, 2018: 7794-7803

[20] $\mathrm{Hu}$ J, Shen L, Sun G. Squeeze-and-excitation networks[C] // Proceedings of the IEEE Conference on Computer Vision and Pattern Recognition. Los Alamitos: IEEE Computer Society Press, 2018: 7132-7141

[21] Woo S, Park J, Lee J Y, et al. CBAM: convolutional block attention module[C] //Proceedings of the European Conference on Computer Vision. Heidelberg: Springer, 2018: 3-19

[22] Wang Yiming, Du Huimin, Zhang Xia, et al. Application of visual attention network in workpiece surface defect detection[J]. Journal of Computer-Aided Design \& Computer Graphics, 2019, 31(9): 1528-1534(in Chinese) (王一鸣, 杜慧敏, 张霞, 等. 视觉注意力网络在工件表面缺 陷检测中的应用 $[\mathrm{J}]$. 计算机辅助设计与图形学学报, 2019, 31(9): 1528-1534)

[23] Li H C, Xiong P F, An J, et al. Pyramid attention network for semantic segmentation[OL]. [2020-08-20]. https://arxiv.org/ pdf/1805.10180.pdf

[24] Badrinarayanan V, Kendall A, Cipolla R. SegNet: a deep convolutional encoder-decoder architecture for image segmentation[J]. IEEE Transactions on Pattern Analysis and Machine Intelligence, 2017, 39(12): 2481-2495

[25] Ronneberger O, Fischer P, Brox T. U-Net: convolutional networks for biomedical image segmentation[C] //Proceedings of the International Conference on Medical Image Computing and Computer-Assisted Intervention. Heidelberg: Springer, 2015: 234-241 